\title{
Die Technikfolgenabschätzung als theoretischer Rahmen
}

Die Technikfolgenabschätzung (TFA) bietet den theoretischen Bezugsrahmen, in den diese Arbeit eingebettet wird. Die Diskussion der TFA soll dazu beitragen, die unterschiedlichen Facetten des Themas aus den verschiedenen Perspektiven $\mathrm{zu}$ analysieren und hieraus ein Leitbild mit Handlungsempfehlungen zu erstellen. Zum besseren Verständnis der Anwendung in vorliegender Arbeit wird die TFA im Folgenden ausführlich erläutert.

\subsection{Definition und Abgrenzung der Technikfolgenabschätzung}

Mitte der 1960er Jahre wurde der Begriff „Technology Assessment“ im US-amerikanischen Kongress geprägt. Treibend hierfür war eine Ungleichheit im Zugang zu entscheidungsrelevanten Informationen zwischen der Legislative und der Exekutive in den USA. So konnte die Exekutive aufgrund ihrer Struktur jederzeit auf die neuesten Informationen zugreifen, während die Legislative diese Möglichkeit nicht hatte und sich zeitlich verzögert über andere Wege informieren musste. Daher drohte ein Machtungleichgewicht in Technikentscheidungen. Etwa zeitgleich geriet der Fortschrittsoptimismus durch Grenzen des Wachstums, Umweltprobleme infolge der Technisierung und durch Diskussionen über militärtechnische Entwicklungen in die Kritik (Grunwald, 2010, S. 65 ff.). Verschiedene Spannungsfelder resultierten aus dem wissenschaftlich-technischen Fortschritt, sodass eine Orientierung verlangt wurde (Grunwald, 2010, S. 41 ff.).

In Deutschland hat sich als Übersetzung von „Technology Assessment“ der Begriff der Technikfolgenabschätzung beziehungsweise Technikbewertung durchgesetzt. Die TFA konnte sich als wissenschaftlich begleiteter Ansatz 
etablieren, um Gesellschaft und Politik bei Einführung einer Technik mittels Reflektion der zu erwartenden Folgen zu unterstützen. Das Ziel der TFA ist nicht die Festlegung, ob eine Technologie sinnvoll ist oder nicht, sondern das Aufzeigen der Auswirkungen, um fundierte Entscheidungen zu ermöglichen. Grunwald beschreibt drei wesentliche konstituierende Elemente, und zwar
„... die Wissenschaftlichkeit auch in Situationen mit hoher Unsicherheit, die systematische Berücksichtigung auch nicht intendierter Folgen und deren unter- schiedliche Wahrnehmung durch Entscheider und Betroffene sowie die Handlungs- und Beratungsorientierung" (Grunwald, 2010, S. 67).

Mit Folgen sind die Auswirkungen menschlicher Entscheidungen im Zusammenhang mit der Anwendung einer Technik gemeint. Diese können beabsichtigt, aber auch zufällig sein. Diese Folgen können mit dem menschlichen Erfahrungswissen allein nicht abgeschätzt werden, sodass hier die Wissenschaftlichkeit über Hypothesenbildung und eine neutrale Bewertung ansetzen muss. Anschließend entsteht ein wechselseitiger Austausch: Gesellschaftliche Perspektiven werden in der Technikfolgenabschätzung (TFA) berücksichtigt, gleichwohl fließen die wissenschaftlichen Ergebnisse zurück an die Beteiligten außerhalb der Wissenschaft (Grunwald, 2010, S. 117 f.).

Grunwald wendet indes ein, dass die TFA keine wissenschaftliche Disziplin darstelle, da über viele Fragen der TFA kein wissenschaftlicher Konsens bestehe und keine allgemein gültige Einführungsliteratur vorliege (Grunwald, 2010, S. 14). Weiters wird das Fehlen einer expliziten Theorie bemängelt, wenngleich implizite theoretische Annahmen und Positionen ausgetauscht werden (Grunwald, 2010, S. 273 f.). Dieses Argument ist zwar durchaus nachvollziehbar, zum Beispiel bezogen auf die diversen Möglichkeiten beim Projektvorgehen (siehe Abschnitt 4.4) und auf den fehlenden begrifflichen Referenzrahmen (Grunwald, 2010, S. 275 f.). Dennoch ist die TFA eine seit gut 50 Jahren institutionell verankerte und gesellschaftlich anerkannte Vorgehensweise, die auf wissenschaftlichen Erkenntnissen fußt (siehe Abschnitt 4.2).

Historisch ist die TFA im Rahmen der wissenschaftlichen Politikberatung entstanden und wird daher oft auf diese reduziert. Jedoch handelt es sich bei Politikberatung und TFA um zwei Verfahren, wie die Gegenüberstellung in Tabelle 4.1 illustriert. 


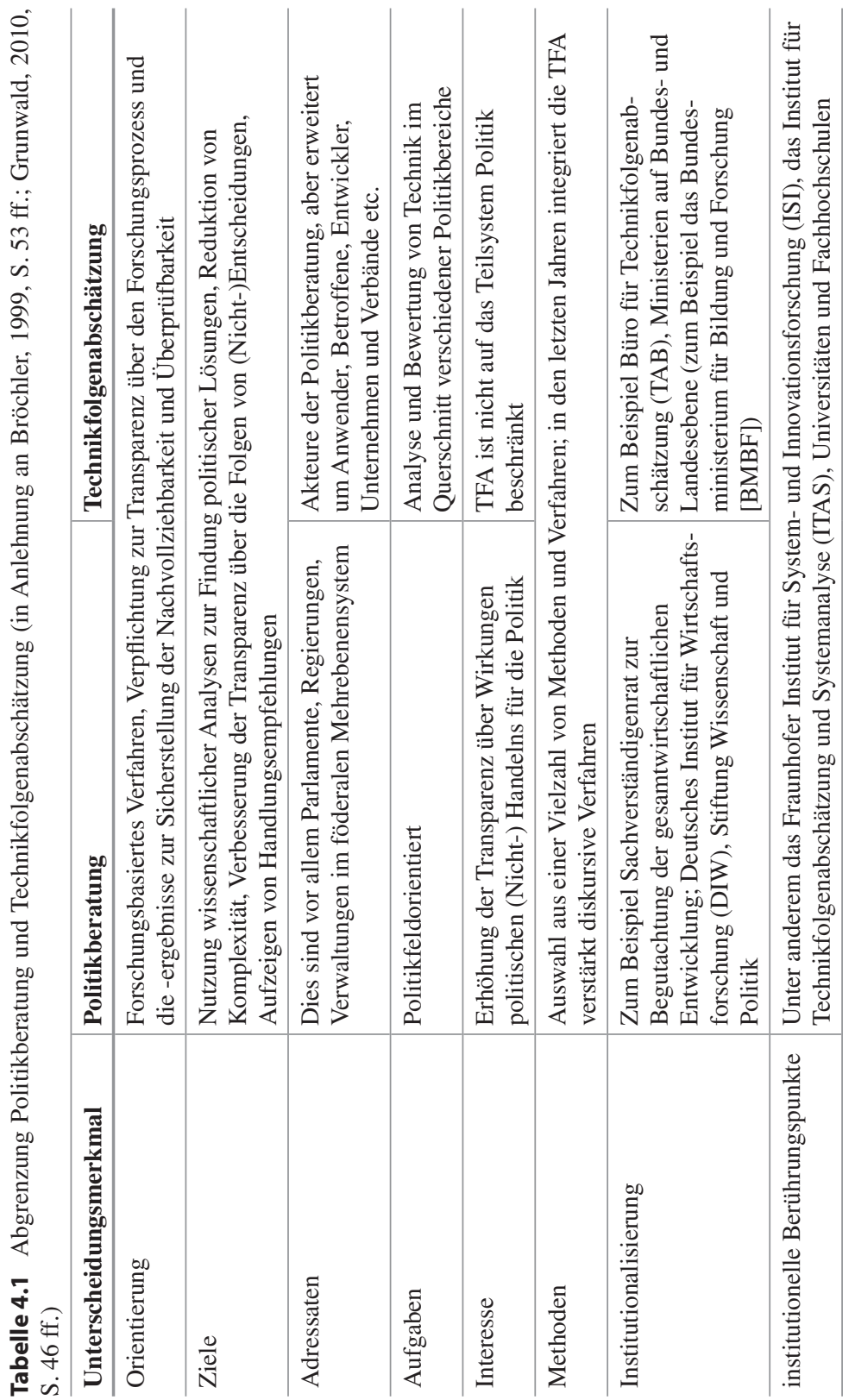


Es zeigt sich, dass Politikberatung und TFA vor allem in der Orientierung, den Zielen, beim Adressaten der Politik und den verwendeten Methoden Gemeinsamkeiten aufweisen. Die TFA lässt sich jedoch von der Politikberatung abgrenzen durch ihren erweiterten Adressatenkreis über die Politik hinaus, ihren querschnittlichen Aufgabenzuschnitt, eine höhere Diskursorientierung und eine institutionelle Verankerung, die eher an der Schnittstelle zwischen Verwaltung und Bürger zu finden ist und weniger im wissenschaftlichen Umfeld (Bröchler, 1999, S. 53 ff.).

Zudem ist die Technikgeneseforschung von der TFA zu unterscheiden. Erstere setzt früher, bereits am Entstehungsprozess von Technologien, an und berücksichtigt auch die Forschung, Entwicklung und Entscheidung bei der Einführung und Anwendung neuer Technologien. Sie kann daher stärkeren Einfluss auf die Ausgestaltung der Technologie nehmen (Zweck, 2013, S. 147).

\subsection{Prägende Institutionen}

Die erste Institution, die sich mit der TFA befasste und später Vorbildcharakter für weitere Einrichtungen hatte, war das Office of Technology Assessment (OTA), welches im Jahr 1972 im US-amerikanischen Kongress gegründet wurde. Das Leitbild war eine zentrale, neutrale und dennoch politiknahe Beratungseinrichtung, die Möglichkeiten und Grenzen wissenschaftlich-technischer Entwicklungen aus einer Hand für den Kongress bearbeiten konnte. Das OTA bestand bis zum Jahr 1995 und hatte zu diesem Zeitpunkt etwa 200 Mitarbeiter aus unterschiedlichen wissenschaftlichen Disziplinen (Grunwald, 2010, S. 67 f.). Seine Hauptaufgaben waren unter anderem der Aufbau einer wissenschaftlichen Beratungskompetenz, Zusammenführung von Informationen für politische Entscheidungsprozesse, Ausarbeitung alternativer Lösungswege und die Abschätzung der hieraus entstehenden Konsequenzen, Einbeziehung externer Experten sowie Integration partizipativer Elemente zur Rückgewinnung des Vertrauens der Öffentlichkeit und Legitimation politischer Entscheidungen (Büllingen, 1999, S. 413 f.). Das OTA publizierte mehr als 700 Studien und zusätzlich Hintergrundpapiere und Workshop-Dokumentationen (Office of Technology Assessment, 2019). Die Schließung der Institution wurde nach den Wahlen 1994 durch die Republikaner aufgrund anderer politischer Prioritäten eingeleitet (Grunwald, 2010, S. 70).

Auf europäischer Ebene ist zum einen das Scientific and Technological Options Assessment (STOA) zu nennen. Es wurde im Jahr 1988 gegründet und ab dem Jahr 1992 in die Verwaltungsstruktur des Europäischen Parlaments integriert 
(European Parliament, 2019). Während vor dem Jahr 2005 vor allem interne Mitarbeitende die Berichterstellung verantworteten, werden seitdem verstärkt Externe eingebunden. Die Rolle der Externen wird nun durch ein europäisches Netzwerk parlamentarischer TFA (European Technology Assessment Group [ETAG]) sowie weitere wissenschaftliche Einrichtungen wahrgenommen. Die Koordination, Festlegung der Gesamtausrichtung sowie Beschließung des Arbeitsprogramms erfolgt dabei durch das STOA-Panel, welches sich entsprechend der Anzahl der parlamentarischen Ausschüsse des europäischen Parlaments in Summe aus 24 Mitgliedern zusammensetzt (Grunwald, 2010, S. 71 f.; Wennrich, 1999b, S. 530 f.).

Zum anderen ist das European Parliamentary Technology Assessment (EPTA)-Network aufzuführen, welches im Jahr 1990 ins Leben gerufen wurde und mittlerweile ein Zusammenschluss von 18 parlamentarischen TFA-Einrichtungen in Europa ist. Entscheidungsorgan ist das EPTA-Council (European Parliamentary Technology Assessment, 2019), welches sich aus Vertretern der jeweiligen Länder zusammensetzt (Grunwald, 2010, S. 73 f.; Wennrich, 1999a, S. 535 f.).

In Deutschland wurde im Jahr 1990 das Büro für TFA beim Deutschen Bundestag (TAB) eingerichtet. Auf Basis einer Ausschreibung wird seine Tätigkeit für jeweils fünf Jahre an eine externe Forschungsinstitution vergeben. Seit Anbeginn wird es vom Institut für Technikfolgenabschätzung und Systemanalyse (ITAS) des Karlsruher Instituts für Technologie (KIT) betrieben, seit 2003 in Kooperation mit dem Fraunhofer Institut für System- und Innovationsforschung in Karlsruhe (ISI). Auftraggeber ist der Ausschuss für Bildung, Forschung und TFA, welcher über Arbeitsschwerpunkte und Projekte des TAB entscheidet (Grunwald, 2010, S. 72). Insgesamt beschäftigen sich in Deutschland etwa 48 Institutionen mit dem Thema TFA (Karlsruher Institut für Technologie, 2019).

\subsection{Forschungs- und Beratungskonzeptionen der TFA}

Vorliegende Arbeit beruht auf einer bestimmten Ausprägung der TFA, von denen es eine ganze Reihe gibt. Die Ursprungsform in den 1970er Jahren wurde wie erläutert durch die Politikberatung geprägt, die dem Staat die Rolle einer zentralen wissenden und steuernden Instanz zuerkannte und die wertfrei, expertenbezogen, systemisch, wissenschaftlich und technikdeterministisch war (Grunwald, 2010, S. 87 ff.). Seitdem gab es Weiterentwicklungen und diverse Ausprägungen. Tabelle 4.2 gibt einen Überblick. 
Tabelle 4.2 Ursprungsform Technikfolgenabschätzung und ihre Weiterentwicklung (in Anlehnung an Grunwald, 2010, S. 87 ff.)

\begin{tabular}{|c|c|c|}
\hline Attribut & Ursprungsform & Weiterentwicklung \\
\hline $\begin{array}{l}\text { Politikberatung } \\
\text { für einen starken } \\
\text { Staat }\end{array}$ & $\begin{array}{l}\text { Ausschließlicher Adressatenkreis } \\
\text { der TFA ist die Politik. Damit } \\
\text { verbunden ist die Sicht auf } \\
\text { den Staat als zentraler Instanz } \\
\text { für Wissensbündelung und } \\
\text { Kompetenz zur Steuerung der } \\
\text { Gesellschaft. }\end{array}$ & $\begin{array}{l}\text { Der Adressatenkreis hat sich } \\
\text { seit den Anfängen erweitert um } \\
\text { zum Beispiel Bürger, Wirtschaft } \\
\text { und Verbände. Dahinter } \\
\text { steht ein stärker dezentrales, } \\
\text { pluralistisches Staatsverständnis. }\end{array}$ \\
\hline Dezisionismus & $\begin{array}{l}\text { Im Fokus der TFA stehen Fakten, } \\
\text { Wertungen bleiben der Politik } \\
\text { vorbehalten. }\end{array}$ & $\begin{array}{l}\text { Die Trennung von Fakten und } \\
\text { Wertungen war nicht durchzu- } \\
\text { halten. Im Austausch zwischen } \\
\text { Experten und Politik entstehen } \\
\text { kognitive und normative Aspekte, } \\
\text { die in der Folge stärker mit- } \\
\text { einander in Einklang gebracht } \\
\text { wurden. }\end{array}$ \\
\hline Experten-TFA & $\begin{array}{l}\text { Es werden lediglich } \\
\text { Experten in die TFA einbezogen. }\end{array}$ & $\begin{array}{l}\text { Um die Sicht der Betroffenen } \\
\text { stärker zu berüicksichtigen } \\
\text { und dem Leitbild der } \\
\text { Demokratisierung gerecht zu } \\
\text { werden, müssen Experten- } \\
\text { analysen um partizipative } \\
\text { Bestandteile ergänzt werden. }\end{array}$ \\
\hline Systemblick & $\begin{array}{l}\text { Der Fokus der TFA liegt } \\
\text { auf einer systemischen } \\
\text { Analyse, da die Folgen von } \\
\text { Technik oft Auswirkungen auf } \\
\text { ein System mit unterschiedlichen } \\
\text { (auch nichtlinearen) Abhängig- } \\
\text { keiten besitzen und daher nicht } \\
\text { auf einzelne Dimensionen } \\
\text { beschränkt werden können. }\end{array}$ & $\begin{array}{l}\text { Dieses Attribut hat nach wie vor } \\
\text { unveränderte Relevanz. }\end{array}$ \\
\hline Szientismus & $\begin{array}{l}\text { Hinter diesem Attribut verbirgt } \\
\text { sich der Anspruch, Technikfolgen } \\
\text { mit allen Variablen vollständig } \\
\text { quantifizieren zu können. }\end{array}$ & $\begin{array}{l}\text { Die Erkenntnis wurde gewonnen, } \\
\text { dass sich gesellschaftliche } \\
\text { Einflüsse und Folgen nicht } \\
\text { mathematisch gestalten lassen. }\end{array}$ \\
\hline $\begin{array}{l}\text { Technikdeter- } \\
\text { minismus }\end{array}$ & $\begin{array}{l}\text { Die vorherrschende Sicht } \\
\text { ist, dass Folgen durch den } \\
\text { Staat modifiziert werden können, } \\
\text { weshalb es eine möglichst genaue } \\
\text { Prognose der Folgen benötigt. } \\
\text { Technikgestaltung spielt keine } \\
\text { Rolle. }\end{array}$ & $\begin{array}{l}\text { Die Bedeutung des sozialen } \\
\text { Prozesses für die Entstehung von } \\
\text { Technik wurde anerkannt und } \\
\text { damit auch die Gestaltungsmög- } \\
\text { lichkeiten in frühen Phasen der } \\
\text { Technikentstehung. }\end{array}$ \\
\hline
\end{tabular}


Im Laufe der Zeit haben sich weitere Verfahren etabliert, die in der Literatur nicht einheitlich benannt werden. Grunwald führt die partizipative TFA, die folgenorientierte TFA, das Vision Assessment, die Technikbewertung und die rationale Technikfolgenbeurteilung auf (Grunwald, 2010, S. 91 ff.). Simonis geht auf die partizipative TFA, die rationale TFA, die strategische und konstruktive TFA, die parlamentarische TFA, die Technikbewertung und Technology Governance ein (Simonis, 2013).

Die Methoden der TFA sind ebenfalls vielfältig. Sie reichen von systemanalytischen Verfahren (zum Beispiel Stoffstromanalyse, Lebenszyklusanalyse, Ökobilanzierung, Risikoanalyse), über prospektive Verfahren (zum Beispiel Szenariotechnik, Delphi-Verfahren), Beteiligungsverfahren (beispielsweise Konsensus-Konferenz, kooperativer Diskurs, Mediationsverfahren), kommunikative Verfahren bis hin zu diskursanalytischen Verfahren (zum Beispiel Interview, Wertbaumverfahren) (Grunwald, 2010, S. 169 f.).

Vorliegende Arbeit zieht die partizipative TFA heran. Unter der partizipativen TFA werden Instrumente und Methoden zusammengefasst, welche die Beteiligung von Interessenvertretern und Laien neben der Beteiligung von Personen aus Wissenschaft und Politik beinhalten. Ziel ist es, die unterschiedlichen gesellschaftlichen Sichtweisen in den Prozess zu integrieren. Hierfür sind insbesondere drei Dimensionen maßgeblich (Abels \& Alfons, 2013, S. 109 f.):

- Die kognitive Dimension und die Beantwortung der Frage, was getan werden kann. Der Adressat ist die Wissenschaft.

- Die normative Dimension und die Beantwortung der Frage, was getan werden darf und soll. Die Adressaten sind das Recht und die Ethik.

- Die voluntative Dimension und die Beantwortung der Frage, was getan werden will. Der Adressat ist die Politik.

An den Dimensionen wird die Vielfalt der Diskussionsebenen deutlich, die mit Blick auf gesellschaftliche Aspekte der TFA integriert werden können. Daran wird auch klar, dass „,weder Politik, noch Recht, Ökonomie, Erziehung oder Ethik [...] je für sich alleine in der Lage [sind], Technik in der funktional differenzierten Gesellschaft zu regulieren“ (Abels \& Alfons, 2013, S. 111).

Es lassen sich grundsätzlich zwei Richtungen der partizipativen TFA unterscheiden: Eine eher wissenschafts- oder expertenorientierte Richtung (,expert stakeholder pTFA“) und eine, welche stärker partizipativ ist, indem Bürgerbeteiligung miteingeschlossen wird (,public pTFA“). In beiden Fällen versuchen 
„... ExpertInnen und Laien, Entscheider und Betroffene, GegnerInnen und BefürworterInnen, gemeinsam zu einem argumentativ begründeten Urteil darüber zu kommen, ob eine umstrittene Technik eingeführt werden soll und wie sie gegebenenfalls zu regulieren ist" (Abels \& Alfons, 2013, S. 110).

Die folgenden Verfahren können den Beteiligungsverfahren zugeordnet werden, über die „pTFA im engeren Sinn“, „Erörterungstermin“, „Konsensus-Konferenz“, „erweiterte Konsensus-Konferenz“, ,Voting Conference“ bis hin zum „Szenario Workshop“ (Abels \& Alfons, 2013, S. 123 ff.). Ebenfalls denkbar sind Diskursverfahren, so zum Beispiel die Diskursanalyse, das Interview oder das Wertbaumverfahren (Grunwald, 2010, S. 185 ff.). Sie alle basieren auf dem Austausch von Argumenten (im Gegensatz zum Verhandeln) und besitzen eher beratenden denn entscheidenden Charakter. Wichtig ist, dass die partizipative TFA strukturell nicht ausreichend in die repräsentative Demokratie eingebunden ist: Sie ermöglicht zwar die Teilnahme am politischen Diskurs und kann Entscheidungen argumentativ vorbereiten, jedoch hat sie (mit Ausnahme des Erörterungstermins) keinen rechtlich wirksamen Einfluss auf das Ergebnis des Verfahrens (Abels \& Alfons, 2013, S. 117 f.). Ziel ist dabei nicht zwingend das Erreichen eines Konsenses (wenngleich Verfahren wie Konsensus-Konferenzen dies suggerieren), sondern die Aufbereitung der Fakten und die Beschreibung einer Vorgehensweise, die das Für und Wider berücksichtigt (Grunwald, 2010, S. 94 f.).

Die Erwartungen an die pTFA sind vielschichtig. Mit ihr wird eine Verbreiterung der Wissensbasis verbunden, da die Perspektiven verschiedener Beteiligter und insbesondere lokales und regionales Wissen aufgegriffen werden können. Gleichermaßen fließen unterschiedliche Wertvorstellungen und Interessen ein, die in der Einschätzung der Technikfolgen positiv und negativ berücksichtigt werden können. Über den Prozess der pTFA kann Technikverständnis ausgebildet werden, was zu einer besseren Entscheidungsfähigkeit, mehr Akzeptanz, einer höheren Legitimation politischer Entscheidungen sowie zur Konfliktvermeidung beziehungsweise -bewältigung führen kann. Insgesamt spricht man der pTFA zu, dass sie über die transparente Darlegung der Bewertungskriterien in einem offenen Prozess einen Beitrag zur Stärkung der Demokratie leisten kann (Grunwald, 2010, S. 91 ff.; Scheffezik, 2003, S. 139).

Die Herausforderungen liegen unter anderem darin, dass über Verfahrensregeln abgesichert wird, dass Beiträge von allen eingebracht werden können und keine Dominanz einzelner Teilnehmer entsteht, dass die Beiträge qualitativ hochwertig sind und die Rolle von Moderatoren und Mediatoren durch die Teilnehmer anerkannt werden. Die Zusammensetzung der Gruppe muss repräsentativ sein, damit die Ergebnisse auch von außen anerkannt werden. Die Ergebnisse selbst 
müssen für die Beteiligten nachvollziehbar sein und - insbesondere im Fall eines unerwarteten Ausgangs - mitgetragen werden. Sofern die pTFA auch auf eine politisch verbindliche Entscheidung hinwirken soll, ist es unabdingbar, dass sie in offizielle Verfahren eingebunden wird (Grunwald, 2010, S. 95 ff.).

\subsection{Projektvorgehen und Methoden}

Für die Strukturierung von Projekten der TFA gibt es in der Literatur mehrere Ansätze. Die Richtlinie 3780 des Verbands Deutscher Ingenieure (VDI) unterscheidet beispielsweise die Phasen der Definition, der Folgenabschätzung, der Bewertung und der Entscheidung (Zweck, 2013, S. 151). Das von der US-amerikanischen Beratungsagentur „Massachusetts Institute Of Technology Research And Engineering“ (MITRE) eingesetzte Vorgehen beinhaltet die Konzeptionsphase, die Systemdefinition, die Potentialabschätzung, die Szenariobildung, die Folgenabschätzung und die Bewertung (Scheffezik, 2003, S. 115 f.). Obwohl die Ansätze hinsichtlich des Detaillierungsgrads voneinander abweichen, unterscheiden sie sich wenig in den Grundelementen und geben gute Anregungen für eine Strukturierung (Grunwald, 2010, S. 122; Scheffezik, 2003, S. 115). Vorliegende Arbeit nimmt diese Anregungen auf und setzt sie in folgende Struktur um:

- Problemdefinition, Beschreibung der zu bewertenden Technologie, der Wirkungsdimensionen und der Akteure (Kapitel 3, 4)

- Beschreibung der Ist-Situation (Kapitel 5, 6)

- Bewertung der Folgen (Kapitel 7, 8, 9)

- Ableitung eines Leitbilds und Analyse politischer Handlungsoptionen (Kapitel 10)

- Allgemeinverständliche Vermittlung der Resultate (Kapitel 11)

\subsection{Wissenschaftliche Herausforderungen}

Verschiedene Verfahren und Methoden kennzeichnen aktuell die TFA, aber auch die Abwesenheit einer übergreifenden, strukturierenden Theorie. Dies erschwert eine trennscharfe Definition. Festgehalten werden kann lediglich, dass die TFA versucht, politischen Entscheidern eine Orientierung zu bieten, indem sie die Möglichkeiten und Grenzen für heute benötigte Entscheidungen durch Reflektion 
auf künftige technische Konsequenzen darlegt (Grunwald, 2010, S. 41). In der Forschungsliteratur mangelt es zudem an einer theoriebasierten empirischen Forschung zur pTFA. Das bedeutet auch, dass bislang mögliche Stärken der pTFA nicht belegt sind (Abels \& Alfons, 2013, S. 119). Bedeutsam für den Erfolg des Einsatzes der pTFA scheint eine ausgewogene Rollenverteilung zwischen Experten und Laien zu sein, mit der sowohl eine hohe Legitimation als auch breite Akzeptanz erzielt werden kann (Abels \& Alfons, 2013, S. 121).

Trotz der genannten Lücken und der damit einhergehenden wissenschaftlichen Herausforderungen bietet die TFA einen geeigneten Rahmen der Bewertung für diese Arbeit. Positiv betrachtet bietet dies einen Interpretations- und kreativen Anwendungsspielraum, der genutzt werden kann.

Im Zentrum der Untersuchung steht die mögliche Öffnung der Daten des öffentlichen Einkaufs. Die Folgen der Öffnung werden mithilfe der Wirkungsdimensionen Strategie, Organisation, Recht, Technologie, Transparenz, Kollaboration und Partizipation untersucht. Diese Dimensionen finden in allen verwendeten Methoden Berücksichtigung: In der Literaturauswertung, der strukturierten Online-Befragung, der Portalanalyse und den Best Practices. Abbildung 4.1 zeigt beispielhafte Aspekte der Vertiefung.

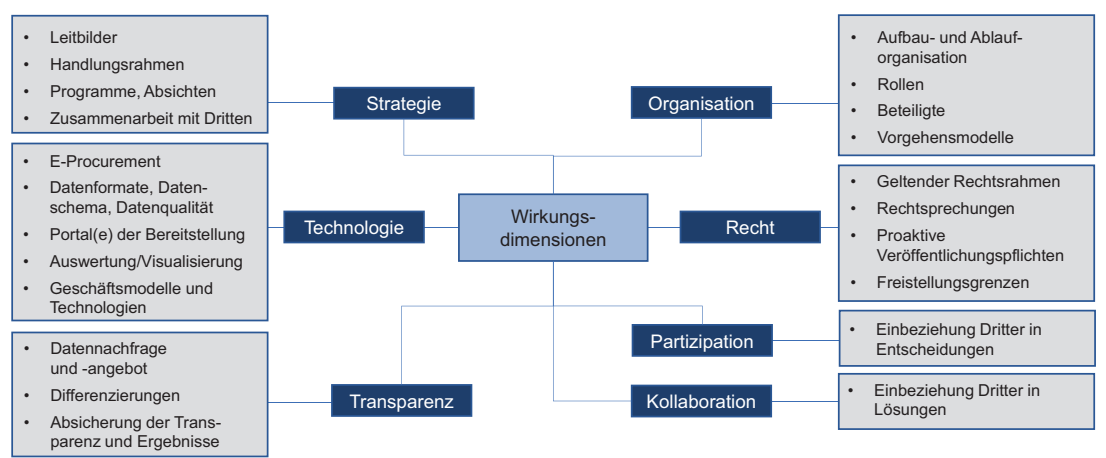

Abbildung 4.1 Wirkungsdimensionen der Analyse und beispielhafte Aspekte der Vertiefung

Unter Einbeziehung der Perspektiven verschiedener Akteure mit unterschiedlichen Erfahrungshintergründen - vor allem im Rahmen der Online-Befragung findet die pTFA Anwendung (Grunwald, 2010, S. 185 f.).

Die befragten Gruppen bestehen aus Vertretern der Wirtschaft, Wissenschaft, Verwaltung, Politik und Medien sowie von Nichtregierungsorganisationen (NGOs), um die Einbeziehung der Bürgerperspektive sicherzustellen. 
Open Access Dieses Kapitel wird unter der Creative Commons Namensnennung 4.0 International Lizenz (http://creativecommons.org/licenses/by/4.0/deed.de) veröffentlicht, welche die Nutzung, Vervielfältigung, Bearbeitung, Verbreitung und Wiedergabe in jeglichem Medium und Format erlaubt, sofern Sie den/die ursprünglichen Autor(en) und die Quelle ordnungsgemäß nennen, einen Link zur Creative Commons Lizenz beifügen und angeben, ob Änderungen vorgenommen wurden.

Die in diesem Kapitel enthaltenen Bilder und sonstiges Drittmaterial unterliegen ebenfalls der genannten Creative Commons Lizenz, sofern sich aus der Abbildungslegende nichts anderes ergibt. Sofern das betreffende Material nicht unter der genannten Creative Commons Lizenz steht und die betreffende Handlung nicht nach gesetzlichen Vorschriften erlaubt ist, ist für die oben aufgeführten Weiterverwendungen des Materials die Einwilligung des jeweiligen Rechteinhabers einzuholen.

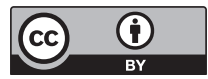

